\title{
Mathematical Model of Schistosomiasis under Flood in Anhui Province
}

\author{
Longxing Qi, ${ }^{1}$ Jing-an Cui, ${ }^{2}$ Tingting Huang, ${ }^{1}$ Fengli Ye, ${ }^{3}$ and Longzhi Jiang ${ }^{4}$ \\ ${ }^{1}$ School of Mathematical Sciences, Anhui University, Hefei 230601, China \\ ${ }^{2}$ College of Science, Beijing University of Civil Engineering and Architecture, Beijing 100044, China \\ ${ }^{3}$ Tongcheng Health Bureau, Tongcheng 231400, China \\ ${ }^{4}$ Tongcheng Schistosomiasis Control Station, Tongcheng 231400, China \\ Correspondence should be addressed to Longxing Qi; qilx@ahu.edu.cn
}

Received 11 January 2014; Accepted 1 February 2014; Published 6 March 2014

Academic Editor: Weiming Wang

Copyright (c) 2014 Longxing Qi et al. This is an open access article distributed under the Creative Commons Attribution License, which permits unrestricted use, distribution, and reproduction in any medium, provided the original work is properly cited.

\begin{abstract}
Based on the real observation data in Tongcheng city, this paper established a mathematical model of schistosomiasis transmission under flood in Anhui province. The delay of schistosomiasis outbreak under flood was considered. Analysis of this model shows that the disease free equilibrium is locally asymptotically stable if the basic reproduction number is less than one. The stability of the unique endemic equilibrium may be changed under some conditions even if the basic reproduction number is larger than one. The impact of flood on the stability of the endemic equilibrium is studied and the results imply that flood can destabilize the system and periodic solutions can arise by Hopf bifurcation. Finally, numerical simulations are performed to support these mathematical results and the results are in accord with the observation data from Tongcheng Schistosomiasis Control Station.
\end{abstract}

\section{Introduction}

As we know, schistosomiasis is a serious water-borne disease. It is not easy to control because of many reasons such as flood. Many reports have shown that flood leads to a serious outbreak of schistosomiasis [1-3]. During the flood period there are a lot of people that come into contact with contaminated water, which may lead to the fact that a lot of people are infected by schistosome [1-3]. In China, Anhui province often encounters floods; in particular in 1998 the flood was one of the most serious flood [1]. Based on the observation data from Tongcheng Schistosomiasis Control Station in Anhui province (Figure 1), we can see that the number of patients and the area of snails increase by a big margin after 1998 in Tongcheng city in Anhui province. Although people know the phenomenon that schistosomiasis will be serious after flood, people do not know the reason and there are only some live reports. Hence, it is necessary to investigate theoretically the effect of flood on the schistosomiasis transmission.
After flood the infected human by cercaria will have an incubation period to become an infectious human. In fact, it is about five weeks from the time of cercaria penetration through skins of human to the time when eggs are discharged [4]. Adult schistosomes in human are capable of producing eggs for a number of years [5]. This leads to breakout of schistosomiasis in many places after flood. For example, the catastrophic flood in 1998 brought a serious impact on the prevalence of schistosomiasis in Anhui province from 1998 to 2000 [1]. Furthermore, the data from Tongcheng Schistosomiasis Control Station (Figure 1) and the report of $\mathrm{Ge}$ et al. [1] both show that schistosomiasis is more serious in three years after flood than in the flood year. This phenomenon is called the delayed effect of flood [1]. In this paper we want to investigate how flood affects the dynamical behavior of schistosomiasis.

Many schistosomiasis models have involved many aspects such as drug-resistant, age-structure, incubation period of snail, and chemotherapy [6-10]. Their results imply that many factors affect the transmission of schistosomiasis. However, 


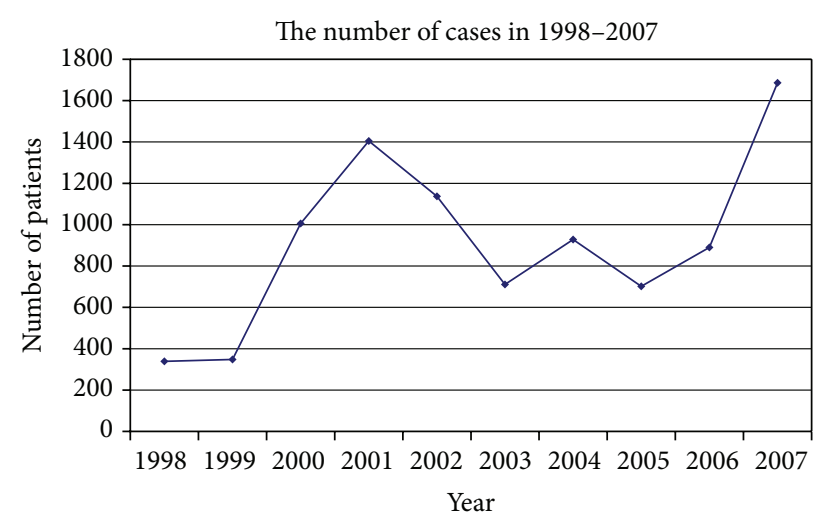

(a)

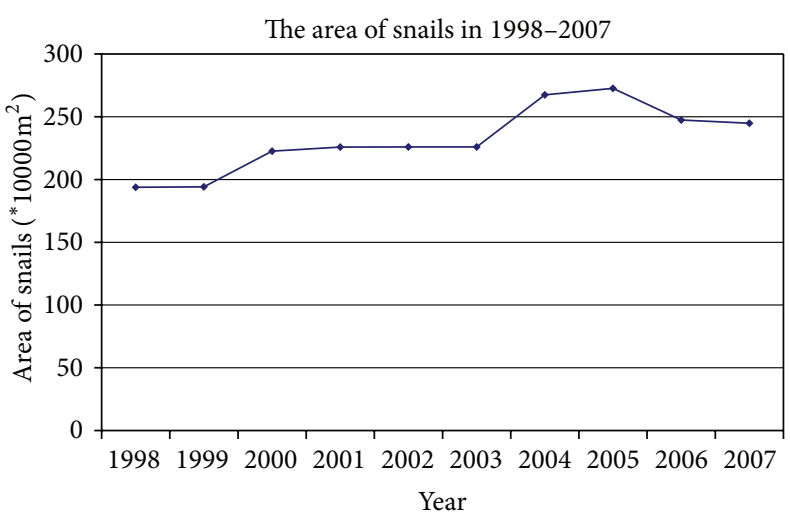

(b)

FIgURE 1: The observation data in Tongcheng city from Tongcheng Schistosomiasis Control Station in 1998-2007.

there are few mathematical models considering the effect of flood in previous papers.

To study the delayed effect of flood, we use a time delay to reflect the incubation period in the infected human. We modified the model in [11]. Distribute human into susceptible $x_{s}(t)$ and infectious $x_{i}(t)$ and snails into susceptible $y_{s}(t)$, preshedding $y_{e}(t)$, and infectious $y_{i}(t)$. The model in [11] reads

$$
\begin{aligned}
& \frac{d x_{s}}{d t}=A_{x}-\mu_{x} x_{s}-\beta_{x} x_{s} y_{i}, \\
& \frac{d x_{i}}{d t}=\beta_{x} x_{s} y_{i}-\left(\mu_{x}+\alpha_{x}\right) x_{i}, \\
& \frac{d y_{s}}{d t}=A_{y}-\mu_{y} y_{s}-\beta_{y} x_{i} y_{s}, \\
& \frac{d y_{e}}{d t}=\beta_{y} x_{i} y_{s}-\left(\mu_{y}+\theta\right) y_{e}, \\
& \frac{d y_{i}}{d t}=\theta y_{e}-\left(\mu_{y}+\alpha_{y}\right) y_{i},
\end{aligned}
$$

where $A_{x}$ is the recruitment rate of human, $\mu_{x}$ is the death rate of human, $\alpha_{x}$ is the disease-induced death rate of human, $\beta_{x}$ is the transmission rate from infectious snails to susceptible human, $A_{y}$ is the recruitment rate of snail host, $\mu_{y}$ is the death rate of snail host, $\alpha_{y}$ is the disease-induced death rate of snail host, $\beta_{y}$ is the transmission rate from infectious human to susceptible snails, and $\theta$ is the translate rate from infected and preshedding snails to shedding snails. In the model, we have studied the stability of equilibria and preferable control strategies.

The goal of this paper is to study the impact of flood on the basic reproduction number and the dynamics of the schistosomiasis transmission. This paper is organized as follows. In Section 2 we establish a schistosomiasis model with a time delay and define the basic reproduction number $R_{0}$. The stability of the disease free equilibrium is obtained in Section 3. We devote Section 4 to the Hopf bifurcation analysis. Section 5 examines mathematical results by numerical simulations.

\section{The Delayed Model}

By incorporating a time delay in human, we have the following model:

$$
\begin{gathered}
\frac{d x_{s}}{d t}=A_{x}-\mu_{x} x_{s}(t)-\beta_{x} x_{s}(t-\tau) y_{i}(t-\tau) e^{-\mu_{x} \tau}, \\
\frac{d x_{i}}{d t}=\beta_{x} x_{s}(t-\tau) y_{i}(t-\tau) e^{-\mu_{x} \tau}-\left(\mu_{x}+\alpha_{x}\right) x_{i}(t), \\
\frac{d y_{s}}{d t}=A_{y}-\mu_{y} y_{s}(t)-\beta_{y} x_{i}(t) y_{s}(t), \\
\frac{d y_{e}}{d t}=\beta_{y} x_{i}(t) y_{s}(t)-\left(\mu_{y}+\theta\right) y_{e}(t), \\
\frac{d y_{i}}{d t}=\theta y_{e}(t)-\left(\mu_{y}+\alpha_{y}\right) y_{i}(t),
\end{gathered}
$$

where $\tau$ is the incubation period in the infected human, that is, the time from cercaria penetration through skins to the time when eggs are discharged.

Define the basic reproduction number according to biological meanings:

$$
R_{0}=\frac{A_{x} A_{y} \theta \beta_{x} e^{-\mu_{x} \tau} \beta_{y}}{\mu_{x} \mu_{y}\left(\mu_{x}+\alpha_{x}\right)\left(\mu_{y}+\alpha_{y}\right)\left(\mu_{y}+\theta\right)} .
$$

These quantities have a clear biological interpretation. Consider the case when an infectious snail is introduced into a purely susceptible people population with size $A_{x} / \mu_{x}$. The size of susceptible people who become infectious people per unit time is $\beta_{x}\left(A_{x} / \mu_{x}\right) \cdot 1 /\left(\mu_{x}+\alpha_{x}\right)$ is the mean infective period of the infectious people and $e^{-\mu_{x} \tau}$ represents the survived rate of people during his infection. On the other hand, infectious people can infect $\beta_{y}\left(A_{y} / \mu_{y}\right)$ susceptible snails which should get through the latent time where the rate of transmission is $\theta$ and then infective period is $1 /\left(\mu_{y}+\alpha_{y}\right)\left(\mu_{y}+\theta\right)$. Thus, $R_{0}$ gives the total number of secondary infectious snails produced by a typical infected snail during its entire period of infectiousness in a completely susceptible population. The following section shows that the 
basic reproduction number $R_{0}$ provides a threshold condition for parasite extinction.

Theorem 1. There exist at most two equilibria:

(i) if $R_{0} \leq 1$, system (2) has a disease free equilibrium $E_{0}=$ $\left(A_{x} / \mu_{x}, 0, A_{y} / \mu_{y}, 0,0\right)$;

(ii) if $R_{0}>1$, system (2) has two equilibria, the disease free equilibrium $E_{0}$ and the unique endemic equilibrium $E=\left(x_{s}^{*}, x_{i}^{*}, y_{s}^{*}, y_{e}^{*}, y_{i}^{*}\right)$, where

$$
\begin{gathered}
x_{s}^{*}=\frac{A_{x}\left(A_{x} \beta_{y}+\mu_{y}\left(\mu_{x}+\alpha_{x}\right)\right)}{\mu_{x}\left(A_{x} \beta_{y}+\mu_{y} R_{0}\left(\mu_{x}+\alpha_{x}\right)\right)}, \\
x_{i}^{*}=\frac{A_{x} \mu_{y}\left(R_{0}-1\right)}{A_{x} \beta_{y}+\mu_{y} R_{0}\left(\mu_{x}+\alpha_{x}\right)}, \\
y_{s}^{*}=\frac{A_{y}\left(A_{x} \beta_{y}+\mu_{y} R_{0}\left(\mu_{x}+\alpha_{x}\right)\right)}{\mu_{y} R_{0}\left(A_{x} \beta_{y}+\mu_{y}\left(\mu_{x}+\alpha_{x}\right)\right)}, \\
y_{e}^{*}=\frac{\mu_{x} \mu_{y}\left(\mu_{x}+\alpha_{x}\right)\left(\mu_{y}+\alpha_{y}\right)\left(R_{0}-1\right)}{\theta \beta_{x} e^{-\mu_{x} \tau}\left(A_{x} \beta_{y}+\mu_{y}\left(\mu_{x}+\alpha_{x}\right)\right)}, \\
y_{i}^{*}=\frac{\mu_{x} \mu_{y}\left(\mu_{x}+\alpha_{x}\right)\left(R_{0}-1\right)}{\beta_{x} e^{-\mu_{x} \tau}\left(A_{x} \beta_{y}+\mu_{y}\left(\mu_{x}+\alpha_{x}\right)\right)} .
\end{gathered}
$$
(2).

Next we will discuss the stabilities of $E_{0}$ and $E$ in system

\section{Stability Analysis of $E_{0}$}

In this section, we will analyze the stability of the disease free equilibrium $E_{0}$ of the delayed model (2) in the two cases: $R_{0}<$ 1 and $R_{0}>1$.

Theorem 2. The disease free equilibrium $E_{0}$ of the system (2) is locally asymptotically stable if $R_{0}<1$ and unstable if $R_{0}>1$.

Proof. Denote $b=\mu_{x}+\alpha_{x}, c=\mu_{y}+\alpha_{y}, d=\mu_{y}+$ $\theta$. By linearizing the system (2) around $E_{0}$ we can obtain the characteristic roots that are $-\mu_{x},-\mu_{y}$ and roots of the following equation:

$$
\begin{aligned}
& \lambda^{3}+(b+c+d) \lambda^{2}+(b c+b d+c d) \lambda \\
& +b c d-\frac{A_{x} A_{y} \theta \beta_{x} e^{-\mu_{x} \tau} \beta_{y}}{\mu_{x} \mu_{y}} e^{-\lambda \tau}=0 .
\end{aligned}
$$

Denote the left-hand side of $(5)$ as $F(\lambda, \tau)$. It is easy to see that

$$
\begin{aligned}
F(0, \tau) & =b c d-\frac{A_{x} A_{y} \theta \beta_{x} e^{-\mu_{x} \tau} \beta_{y}}{\mu_{x} \mu_{y}} e^{-\lambda \tau} \\
& =b c d\left(1-R_{0}\right)
\end{aligned}
$$

$$
\begin{gathered}
F_{\lambda}^{\prime}(\lambda, \tau)=3 \lambda^{2}+2(b+c+d) \lambda+(b c+b d+c d) \\
+\tau \frac{A_{x} A_{y} \theta \beta_{x} e^{-\mu_{x} \tau} \beta_{y}}{\mu_{x} \mu_{y}} e^{-\lambda \tau} .
\end{gathered}
$$

(i) If $R_{0}>1, F(0, \tau)<0, F_{\lambda}^{\prime}(\lambda, \tau)>0$ for $\lambda \geq 0$ and $\tau>0$. Thus, (5) has a positive real solution for $\tau>0$ and the disease free equilibrium $E_{0}$ is unstable.

(ii) If $R_{0}<1, F(0, \tau)>0$. Since $F_{\lambda}^{\prime}(\lambda, \tau)>0$ for $\lambda \geq 0$ and $\tau>0$, (5) does not have nonnegative real roots for $\tau>0$. Hence, if (5) has roots with nonnegative real parts they must be complex roots. Moreover these complex roots should be obtained from a pair of complex conjugate roots crossing the imaginary axis. Thus, (5) must have a pair of purely imaginary roots $\lambda= \pm \omega i$ for some $\tau>0$. Without loss of generality we assume that $\omega>0$. Then $\omega$ must be a positive solution of the following equation:

$$
\begin{array}{r}
-\omega^{3} i-(b+c+d) \omega^{2}+(b c+b d+c d) \omega i+b c d \\
-\frac{A_{x} A_{y} \theta \beta_{x} e^{-\mu_{x} \tau} \beta_{y}}{\mu_{x} \mu_{y}}(\cos (\omega \tau)-i \sin (\omega \tau))=0,
\end{array}
$$

which is equivalent to

$$
-\omega^{3}+(b c+b d+c d) \omega
$$

$$
+\frac{A_{x} A_{y} \theta \beta_{x} e^{-\mu_{x} \tau} \beta_{y}}{\mu_{x} \mu_{y}} \sin (\omega \tau)=0,
$$$$
-(b+c+d) \omega^{2}+b c d
$$

$$
-\frac{A_{x} A_{y} \theta \beta_{x} e^{-\mu_{x} \tau} \beta_{y}}{\mu_{x} \mu_{y}} \cos (\omega \tau)=0 .
$$

Let $A_{x} A_{y} \theta \beta_{x} e^{-\mu_{x} \tau} \beta_{y} / \mu_{x} \mu_{y}=e$. Hence,

$$
\begin{aligned}
\omega^{6}+ & \left(b^{2}+c^{2}+d^{2}\right) \omega^{4}+\left(b^{2} c^{2}+b^{2} d^{2}+c^{2} d^{2}\right) \omega^{2} \\
& +\left(b^{2} c^{2} d^{2}-e^{2}\right)=0 .
\end{aligned}
$$

Assuming $z=\omega^{2}$, we can obtain

$$
z^{3}+\alpha z^{2}+\beta z+\gamma=0
$$

where $\alpha=b^{2}+c^{2}+d^{2}>0, \beta=b^{2} c^{2}+b^{2} d^{2}+c^{2} d^{2}>0, \gamma=$ $b^{2} c^{2} d^{2}-e^{2}>0$ as $R_{0}<1$.

From [12, Lemma 3.31], if $\alpha \geq 0, \beta>0, \gamma \geq 0$, then (10) has no positive real roots. This implies that (7) does not have positive solution $\omega$ since $R_{0}<1$. Therefore, (5) does not have purely imaginary roots. Consequently, the real parts of all eigenvalues of $E_{0}$ are negative for all positive $\tau$. This indicates that the disease free equilibrium $E_{0}$ is locally asymptotically stable if $R_{0}<1$. 


\section{Hopf Bifurcation Analysis}

In this section, we turn to the study of the stability of the endemic equilibrium $E$ when $R_{0}>1$. Notice that $R_{0}>1$ is equivalent to

$$
\tau<\tau^{*}=\frac{1}{\mu_{x}} \ln \frac{A_{x} A_{y} \theta \beta_{x} \beta_{y}}{\mu_{x} \mu_{y}\left(\mu_{x}+\alpha_{x}\right)\left(\mu_{y}+\alpha_{y}\right)\left(\mu_{y}+\theta\right)} .
$$

The characteristic equation of $E$ is

$$
\begin{aligned}
\lambda^{5}+ & a_{1} \lambda^{4}+a_{2} \lambda^{3}+a_{3} \lambda^{2}+a_{4} \lambda+a_{5} \\
& =e^{-\lambda \tau}\left(b_{1} \lambda^{4}+b_{2} \lambda^{3}+b_{3} \lambda^{2}+b_{4} \lambda+b_{5}\right),
\end{aligned}
$$

where

$$
\begin{gathered}
a_{1}=\mu_{x}+\mu_{y}+b+c+d>0 \\
a_{2}=b c+b d+b \mu_{x}+b \mu_{y}+c d+c \mu_{x}+c \mu_{y} \\
+d \mu_{x}+d \mu_{y}+\mu_{x} \mu_{y}>0 \\
a_{3}=b c d+b c \mu_{x}+b c \mu_{y}+c d \mu_{x} \\
+c d \mu_{y}+d \mu_{x} \mu_{y} \\
a_{4}=b c d \mu_{x}+b c d \mu_{y}+c d \mu_{x} \mu_{y} \\
a_{5}=b c d \mu_{x} \mu_{y} \\
b_{1}=-\beta_{x} y_{i}^{*} e^{-\mu_{x} \tau}<0 \\
b_{3}=-\beta_{x} y_{i}^{*} e^{-\mu_{x} \tau}\left(b \mu_{y}+b d+d \mu_{y}+b c+c d+c \mu_{y}\right)+b c d \\
b_{4}=-\beta_{x} y_{i}^{*} e^{-\mu_{x} \tau}\left(b d \mu_{y}+b c \mu_{y}+c d \mu_{y}+b c d\right) \\
+b c d\left(\mu_{x}+\mu_{y}\right) \\
b_{5}=-\beta_{x} y_{i}^{*} e^{-\mu_{x} \tau} b c d \mu_{y}+b c d \mu_{x} \mu_{y} .
\end{gathered}
$$

In the following, it can be shown that (12) does not have nonnegative real roots for $\tau>0$. Let

$$
\begin{gathered}
\tilde{a}_{3}=a_{3}-b c d e^{-\lambda \tau}, \\
\tilde{a}_{4}=a_{4}-b c d\left(\mu_{x}+\mu_{y}\right) e^{-\lambda \tau}, \\
\tilde{a}_{5}=a_{5}-b c d \mu_{x} \mu_{y} e^{-\lambda \tau}, \\
\tilde{b}_{3}=-\beta_{x} y_{i}^{*} e^{-\mu_{x} \tau}\left(b \mu_{y}+b d+d \mu_{y}+b c+c d+c \mu_{y}\right)<0, \\
\tilde{b}_{4}=-\beta_{x} y_{i}^{*} e^{-\mu_{x} \tau}\left(b d \mu_{y}+b c \mu_{y}+c d \mu_{y}+b c d\right)<0, \\
\tilde{b}_{5}=-\beta_{x} y_{i}^{*} e^{-\mu_{x} \tau} b c d \mu_{y}<0 .
\end{gathered}
$$

Note that $\tilde{a}_{3}>0, \tilde{a}_{4}>0, \tilde{a}_{5}>0$ for all $\lambda \geq 0$ and $\tau>0$. We rewrite (12) in the following form:

$$
\begin{aligned}
\lambda^{5}+ & a_{1} \lambda^{4}+a_{2} \lambda^{3}+\tilde{a}_{3} \lambda^{2}+\tilde{a}_{4} \lambda+\tilde{a}_{5} \\
& =e^{-\lambda \tau}\left(b_{1} \lambda^{4}+b_{2} \lambda^{3}+\tilde{b}_{3} \lambda^{2}+\tilde{b}_{4} \lambda+\tilde{b}_{5}\right) .
\end{aligned}
$$

It is easy to see that the left-hand side in (15) is positive while the right-hand side is negative for all $\lambda \geq 0$. Then (12) does not have nonnegative real solutions. Now we consider whether or not (12) has purely imaginary solutions.

Suppose $\lambda=\omega i, \omega>0$ for some $\tau>0$, is a root of (12). Then we have

$$
\begin{aligned}
\omega^{5} i+ & a_{1} \omega^{4}-a_{2} \omega^{3} i-a_{3} \omega^{2}+a_{4} \omega i+a_{5} \\
= & {[\cos (\omega \tau)-i \sin (\omega \tau)] } \\
& \times\left(b_{1} \omega^{4}-b_{2} \omega^{3} i-b_{3} \omega^{2}+b_{4} \omega i+b_{5}\right) .
\end{aligned}
$$

Therefore

$$
\begin{aligned}
\omega^{5}-a_{2} \omega^{3}+a_{4} \omega= & \cos (\omega \tau)\left(-b_{2} \omega^{3}+b_{4} \omega\right) \\
& -\sin (\omega \tau)\left(b_{1} \omega^{4}-b_{3} \omega^{2}+b_{5}\right), \\
a_{1} \omega^{4}-a_{3} \omega^{2}+a_{5}= & \cos (\omega \tau)\left(b_{1} \omega^{4}-b_{3} \omega^{2}+b_{5}\right) \\
& +\sin (\omega \tau)\left(-b_{2} \omega^{3}+b_{4} \omega\right) .
\end{aligned}
$$

From (17), we obtain

$$
\begin{aligned}
& \left(a_{1} \omega^{4}-a_{3} \omega^{2}+a_{5}\right)^{2}+\left(\omega^{5}-a_{2} \omega^{3}+a_{4} \omega\right)^{2} \\
& =\left(b_{1} \omega^{4}-b_{3} \omega^{2}+b_{5}\right)^{2}+\left(-b_{2} \omega^{3}+b_{4} \omega\right)^{2} ;
\end{aligned}
$$

that is,

$$
\begin{gathered}
\omega^{10}+\left(a_{1}^{2}-2 a_{2}-b_{1}^{2}\right) \omega^{8}+\left(a_{2}^{2}+2 a_{4}-2 a_{1} a_{3}\right. \\
\left.-b_{2}^{2}+2 b_{1} b_{3}\right) \omega^{6} \\
+\left(a_{3}^{2}-2 a_{2} a_{4}+2 a_{1} a_{5}+2 b_{2} b_{4}-b_{3}^{2}-2 b_{1} b_{5}\right) \omega^{4} \\
+\left(a_{4}^{2}-2 a_{3} a_{5}-b_{4}^{2}+2 b_{3} b_{5}\right) \omega^{2}+\left(a_{5}^{2}-b_{5}^{2}\right)=0 .
\end{gathered}
$$

Let $z=\omega^{2}$ again; we obtain

$$
z^{5}+c_{1} z^{4}+c_{2} z^{3}+c_{3} z^{2}+c_{4} z+c_{5}=0,
$$

where

$$
\begin{gathered}
c_{1}=a_{1}^{2}-2 a_{2}-b_{1}^{2}, \\
c_{2}=a_{2}^{2}+2 a_{4}-2 a_{1} a_{3}-b_{2}^{2}+2 b_{1} b_{3}, \\
c_{3}=a_{3}^{2}-2 a_{2} a_{4}+2 a_{1} a_{5}+2 b_{2} b_{4}-b_{3}^{2}-2 b_{1} b_{5}, \\
c_{4}=a_{4}^{2}-2 a_{3} a_{5}-b_{4}^{2}+2 b_{3} b_{5}, \\
c_{5}=a_{5}^{2}-b_{5}^{2} .
\end{gathered}
$$


Because (20) is very complex, the roots cannot easily be found. However, we know there are positive roots in some conditions. For example, if $c_{5}<0$, then (20) has at least a positive root, say $z_{0}$, and (19) has at least a positive root $\omega_{0}=\sqrt{z_{0}}$. Consequently, the endemic equilibrium $E$ may lose stability and lead to oscillations because the time delay $\tau>0$. In this case, we will do bifurcation analysis by $\tau$ as bifurcation parameter in the following.

Let $\lambda(\tau)=\xi(\tau)+i \omega(\tau)$ be a root of (12) such that $\xi\left(\tau_{0}\right)=0, \omega\left(\tau_{0}\right)=\omega_{0}\left(\omega_{0}>0\right)$ for some initial value of the bifurcation parameter $\tau_{0}$. From (17) we can obtain

$$
\begin{aligned}
\tau_{j} & \frac{1}{\omega_{0}} \arccos ( \\
& \left(\left(a_{1} b_{1}-b_{2}\right) \omega_{0}^{8}+\left(b_{4}+a_{2} b_{2}-a_{1} b_{3}-a_{3} b_{1}\right) \omega_{0}^{6}\right. \\
& \left.+\left(-a_{2} b_{4}-a_{4} b_{2}+a_{1} b_{5}+a_{3} b_{3}+a_{5} b_{1}\right) \omega_{0}^{4}\right) \\
& \times\left(\left(b_{1} \omega_{0}^{4}-b_{3} \omega_{0}^{2}+b_{5}\right)^{2}+\left(-b_{2} \omega_{0}^{3}+b_{4} \omega_{0}\right)^{2}\right)^{-1} \\
& \left.+\frac{\left(a_{4} b_{4}-a_{3} b_{5}-a_{5} b_{3}\right) \omega_{0}^{2}+a_{5} b_{5}}{\left(b_{1} \omega_{0}^{4}-b_{3} \omega_{0}^{2}+b_{5}\right)^{2}+\left(-b_{2} \omega_{0}^{3}+b_{4} \omega_{0}\right)^{2}}\right) \\
+\frac{2 j \pi}{\omega_{0}}, \quad j= & 0,1,2, \ldots
\end{aligned}
$$

Now we can show the transversal condition $\left.(d \operatorname{Re} \lambda(\tau) / d \tau)\right|_{\tau=\tau_{0}} \neq 0$.

Differentiating (12) with respect to $\tau$ yields

$$
\begin{aligned}
\left(5 \lambda^{4}+\right. & 4 a_{1} \lambda^{3}+3 a_{2} \lambda^{2}+2 a_{3} \lambda+a_{4}+\tau e^{-\lambda \tau} \\
& \times\left(b_{1} \lambda^{4}+b_{2} \lambda^{3}+b_{3} \lambda^{2}+b_{4} \lambda+b_{5}\right) \\
& \left.-e^{-\lambda \tau}\left(4 b_{1} \lambda^{3}+3 b_{2} \lambda^{2}+2 b_{3}+b_{4}\right)\right) \frac{d \lambda}{d \tau} \\
= & -\lambda e^{-\lambda \tau}\left(b_{1} \lambda^{4}+b_{2} \lambda^{3}+b_{3} \lambda^{2}+b_{4} \lambda+b_{5}\right) .
\end{aligned}
$$

Using (12), we obtain

$$
\begin{aligned}
\left(\frac{d \lambda}{d \tau}\right)^{-1}= & \left(5 \lambda^{4}+4 a_{1} \lambda^{3}+3 a_{2} \lambda^{2}+2 a_{3} \lambda+a_{4}\right. \\
& +\tau e^{-\lambda \tau}\left(b_{1} \lambda^{4}+b_{2} \lambda^{3}+b_{3} \lambda^{2}+b_{4} \lambda+b_{5}\right) \\
& \left.-e^{-\lambda \tau}\left(4 b_{1} \lambda^{3}+3 b_{2} \lambda^{2}+2 b_{3}+b_{4}\right)\right) \\
& \times\left(-\lambda e^{-\lambda \tau}\left(b_{1} \lambda^{4}+b_{2} \lambda^{3}+b_{3} \lambda^{2}+b_{4} \lambda+b_{5}\right)\right)^{-1} \\
= & \frac{5 \lambda^{4}+4 a_{1} \lambda^{3}+3 a_{2} \lambda^{2}+2 a_{3} \lambda+a_{4}}{-\lambda\left(\lambda^{5}+a_{1} \lambda^{4}+a_{2} \lambda^{3}+a_{3} \lambda^{2}+a_{4} \lambda+a_{5}\right)} \\
& +\frac{4 b_{1} \lambda^{3}+3 b_{2} \lambda^{2}+2 b_{3}+b_{4}}{\lambda\left(b_{1} \lambda^{4}+b_{2} \lambda^{3}+b_{3} \lambda^{2}+b_{4} \lambda+b_{5}\right)}-\frac{\tau}{\lambda} .
\end{aligned}
$$

Then,

$$
\begin{aligned}
& \operatorname{sign}\left\{\frac{d \operatorname{Re} \lambda}{d \tau}\right\}_{\lambda=i \omega_{0}} \\
& =\operatorname{sign}\left\{\operatorname{Re}\left(\frac{d \lambda}{d \tau}\right)^{-1}\right\}_{\lambda=i \omega_{0}} \\
& =\operatorname{sign}\left\{\operatorname{Re}\left[\frac{5 \lambda^{4}+4 a_{1} \lambda^{3}+3 a_{2} \lambda^{2}+2 a_{3} \lambda+a_{4}}{-\lambda\left(\lambda^{5}+a_{1} \lambda^{4}+a_{2} \lambda^{3}+a_{3} \lambda^{2}+a_{4} \lambda+a_{5}\right)}\right]_{\lambda=i \omega_{0}}\right. \\
& \left.+\operatorname{Re}\left[\frac{4 b_{1} \lambda^{3}+3 b_{2} \lambda^{2}+2 b_{3}+b_{4}}{\lambda\left(b_{1} \lambda^{4}+b_{2} \lambda^{3}+b_{3} \lambda^{2}+b_{4} \lambda+b_{5}\right)}\right]_{\lambda=i \omega_{0}}\right\} \\
& =\operatorname{sign}\left\{\operatorname { R e } \left[\left(\left(-2 a_{3} \omega_{0}+4 a_{1} \omega_{0}^{3}\right)\right.\right.\right. \\
& \left.+\left(-3 a_{2} \omega_{0}^{2}+5 \omega_{0}^{4}+a_{4}\right) i\right) \\
& \times\left(\omega _ { 0 } \left(\left(a_{1} \omega_{0}^{4}-a_{3} \omega_{0}^{2}+a_{5}\right)\right.\right. \\
& \left.\left.\left.+\left(\omega_{0}^{5}-a_{2} \omega_{0}^{3}+a_{4} \omega_{0}\right) i\right)\right)^{-1}\right] \\
& \left.+\operatorname{Re}\left[\frac{\left(2 b_{3} \omega_{0}-4 b_{1} \omega_{0}^{3}\right)+\left(3 b_{2} \omega_{0}^{2}-b_{4}\right) i}{\omega_{0}\left(\left(b_{1} \omega_{0}^{4}+b_{5}-b_{3} \omega_{0}^{2}\right)+\left(b_{4} \omega_{0}-b_{2} \omega_{0}^{3}\right) i\right)}\right]\right\} \\
& =\operatorname{sign}\left\{\left(5 \omega_{0}^{8}+\left(4 a_{1}^{2}-8 a_{2}-4 b_{1}^{2}\right) \omega_{0}^{6}\right.\right. \\
& +\left(3 a_{2}^{2}-6 a_{1} a_{3}+6 a_{4}+6 b_{1} b_{3}-3 b_{2}^{2}\right) \omega_{0}^{4} \\
& +\left(2 a_{3}^{2}-4 a_{2} a_{4}+4 a_{1} a_{5}+4 b_{2} b_{4}-2 b_{3}^{2}-4 b_{1} b_{5}\right) \omega_{0}^{2} \\
& \left.+\left(a_{4}^{2}-2 a_{3} a_{5}+2 b_{3} b_{5}-b_{4}^{2}\right)\right) \\
& \times\left(\left(a_{1} \omega_{0}^{4}-a_{3} \omega_{0}^{2}+a_{5}\right)^{2}\right. \\
& \left.\left.+\left(\omega_{0}^{5}-a_{2} \omega_{0}^{3}+a_{4} \omega_{0}\right)^{2}\right)^{-1}\right\} .
\end{aligned}
$$

If we denote $z_{0}=\omega_{0}^{2}$, we get

$$
\begin{aligned}
\operatorname{sign} & \left\{\frac{d \operatorname{Re} \lambda}{d \tau}\right\}_{\lambda=i \omega_{0}} \\
& =\operatorname{sign}\left\{\frac{5 z_{0}^{4}+4 c_{1} z_{0}^{3}+3 c_{2} z_{0}^{2}+2 c_{3} z_{0}+c_{4}}{\left(a_{1} \omega_{0}^{4}-a_{3} \omega_{0}^{2}+a_{5}\right)^{2}+\left(\omega_{0}^{5}-a_{2} \omega_{0}^{3}+a_{4} \omega_{0}\right)^{2}}\right\} .
\end{aligned}
$$

Denote $f(z)=z^{5}+c_{1} z^{4}+c_{2} z^{3}+c_{3} z^{2}+c_{4} z+c_{5}$. Suppose $\omega_{0}$ is the largest positive simple root of (19); from [12, Lemma 3.32 and Theorem 3.32], we have

$$
\left.\frac{d f(z)}{d z}\right|_{z=z_{0}}=5 z_{0}^{4}+4 c_{1} z_{0}^{3}+3 c_{2} z_{0}^{2}+2 c_{3} z_{0}+c_{4}>0 .
$$




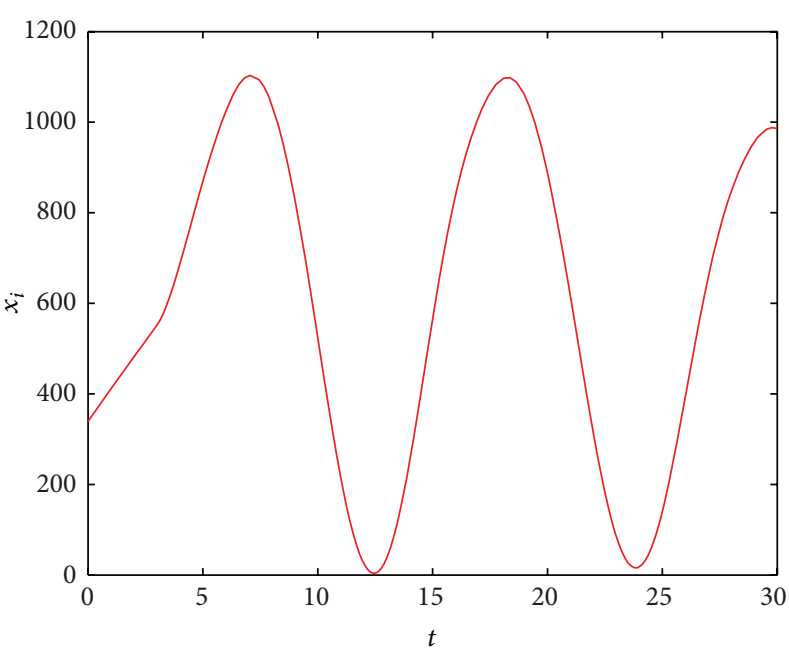

(a) The trajectory of $x_{i}$



(b) The trajectory of $y_{i}$

FIGURE 2: The trajectories of $x_{i}$ and $y_{i}$ occur oscillations when $\tau_{0}=3$.

Thus,

$$
\begin{aligned}
& \operatorname{sign}\left\{\frac{d \operatorname{Re} \lambda}{d \tau}\right\}_{\lambda=i \omega_{0}} \\
&=\operatorname{sign}\left\{\left(\left.\frac{d f(z)}{d z}\right|_{z=z_{0}}\right)\right. \\
& \quad \times\left(\left(a_{1} \omega_{0}^{4}-a_{3} \omega_{0}^{2}+a_{5}\right)^{2}\right. \\
&\left.\left.\quad+\left(\omega_{0}^{5}-a_{2} \omega_{0}^{3}+a_{4} \omega_{0}\right)^{2}\right)^{-1}\right\}=+1 .
\end{aligned}
$$

Summarizing the above analysis, we have the following results.

Theorem 3. If $R_{0}>1, c_{5}<0$ and $\omega_{0}$ is the largest positive simple root of (19), a Hopf bifurcation occurs around the endemic equilibrium $E$ of the delayed model (2).

\section{Numerical Simulations}

Based on the observation data from the investigation of Tongcheng Schistosomiasis Control Station in Anhui province, we estimated transmission rates in our model. Also according to the previous papers $[7-9,11,13]$, we choose the parameter values in Table 1 . Thus, $R_{0}>1, \tau^{*}=327$, and $c_{5}<0$ when $\tau=0.1$.

Note that the bifurcation parameter $\tau_{0}=3$ at this time. We performed some simulations and obtained Figure 2. From Figure 2 we can see that Hopf bifurcation can occur when $\tau_{0}=3$. This implies that schistosomiasis will break out in about three years after flood. It is also in accord with the investigation of Tongcheng Schistosomiasis Control Station. This phenomenon is also in accord with the report of the whole Anhui province [1]. From our theoretical results and
TABLE 1: Values of parameters.

\begin{tabular}{lcc}
\hline Parameters & Values (per capita per year) & References \\
\hline$A_{x}$ & 6 & {$[8,9]$} \\
$\mu_{x}$ & 0.014 & {$[8,9,11]$} \\
$\alpha_{x}$ & $10^{-5}$ & {$[8,9]$} \\
$\beta_{x}$ & 0.003 & Estimated \\
$A_{y}$ & 100 & {$[8,9]$} \\
$\mu_{y}$ & 0.3 & {$[8,9,11]$} \\
$\alpha_{y}$ & 0.01 & {$[9]$} \\
$\beta_{y}$ & 0.001 & Estimated \\
$\theta$ & 9.125 & {$[13]$} \\
\hline
\end{tabular}

the reports we can see that schistosomiasis will break out in about the third year after a flood. Hence, we can get the result that the delayed effect of flood may be caused by the incubation period of schistosome in the infected human.

\section{Discussion}

In this paper, based on the observation data in Tongcheng Schistosomiasis Control Station in Anhui province we have modified our previous model by including a time delay that describes the incubation period of schistosome within infected human. We define the basic reproduction number $R_{0}$ according to biological meanings and give the existence of the disease free equilibrium and the endemic equilibrium. We find that, if $R_{0}<1$, then the disease free equilibrium is locally asymptotically stable. However, the stability of the unique endemic equilibrium may be changed under some condition even if the basic reproduction number is larger than one. The results imply that the time delay can destabilize the system and periodic solutions can arise by Hopf bifurcation.

Numerical simulations imply that schistosomiasis will break out in about three years after flood. Furthermore the 
observation data show that schistosomiasis will be the most serious in about the third year after flood. From Figure 1, we can see that the number of patients and snails did not greatly change in 1998 and 1999. However, in 2001 the number of patients became about 5 times that of 1998, and the area of snails became about two times that of 1998. In our simulations, there is a little difference. Our results are higher than the observation data. We think the reason may be that after flood the government dispatched a large number of manpower and material resources to control the spread of the disease. In summary, our theoretical results are in accord with the investigation of Tongcheng Schistosomiasis Control Station and the report of Anhui Province Institute of Schistosomiasis for the whole Anhui province. Hence, we can obtain the result that after flood the delayed effect of flood may be caused by the incubation period of schistosome in the definitive host. Furthermore, the period of outbreak is about three years after flood in Anhui province.

\section{Conflict of Interests}

The authors declare that there is no conflict of interests regarding the publication of this paper.

\section{Acknowledgments}

This research is supported by National Natural Science Foundation of China (11126177 and 11071011), Natural Science Foundation of Anhui Province (1208085QA15), and the Foundation for Young Talents in College of Anhui Province (2012SQRL021), as well as National Scholarship Foundation of China, Funding Project for Academic Human Resources Development in Institutions of Higher Learning under the Jurisdiction of Beijing Municipality (PHR201107123), Doctoral Fund of Ministry of Education of China (20113401110001, 20103401120002), the Key Natural Science Foundation of the Anhui Higher Education Institutions of China (KJ2009A49), and Students Research Training Program of Anhui University. The authors would like to thank anonymous reviewers for very helpful suggestions which improved greatly this paper.

\section{References}

[1] J. H. Ge, S. Q. Zhang, T. P. Wang et al., "Efects of flood on the prevalence of schistosomiasis in Anhui province in 1998," Journal of Tropical Diseases and Parasitology, vol. 2, pp. 131-134, 2004.

[2] S. B. Mao, Biology of Schistosome and Control of Schistosomiasis, People's Health Press, Beijing, China, 1990.

[3] X. N. Zhou, J. G. Guo, X. H. Wu et al., "Epedemiology of schistosomiasis in the people's republic of China," Emerging Infectious Diseases, vol. 13, no. 10, pp. 1470-1476, 2004.

[4] C. Castillo-Chavez, Z. Feng, and D. Xu, "A schistosomiasis model with mating structure and time delay," Mathematical Biosciences, vol. 211, no. 2, pp. 333-341, 2008.

[5] A. D. Barbour, "Modeling the transmission of schistosomiasis: an introductory view," The American Journal of Tropical Medicine and Hygiene, vol. 55, no. 5, pp. 135-143, 1996.
[6] D. Cioli, "Chemotherapy of schistosomiasis: an update," Parasitology Today, vol. 14, no. 10, pp. 418-422, 1998.

[7] Z. Feng, J. Curtis, and D. J. Minchella, "The influence of drug treatment on the maintenance of schistosome genetic diversity," Journal of Mathematical Biology, vol. 43, no. 1, pp. 52-68, 2001.

[8] Z. Feng, A. Eppert, F. A. Milner, and D. J. Minchella, "Estimation of parameters governing the transmission dynamics of schistosomes," Applied Mathematics Letters, vol. 17, no. 10, pp. 1105-1112, 2004.

[9] Z. Feng, C.-C. Li, and F. A. Milner, "Schistosomiasis models with two migrating human groups," Mathematical and Computer Modelling, vol. 41, no. 11-12, pp. 1213-1230, 2005.

[10] S. Liang, D. Maszle, and R. C. Spear, "A quantitative framework for a multi-group model of schistosomiasis japonicum transmission dynamics and control in Sichuan, China," Acta Tropica, vol. 82, no. 2, pp. 263-277, 2002.

[11] L. Qi, J.-A. Cui, Y. Gao, and H. Zhu, "Modeling the schistosomiasis on the islets in Nanjing," International Journal of Biomathematics, vol. 5, no. 4, Article ID 1250037, 17 pages, 2012.

[12] H.-M. Wei, X.-Z. Li, and M. Martcheva, "An epidemic model of a vector-borne disease with direct transmission and time delay," Journal of Mathematical Analysis and Applications, vol. 342, no. 2, pp. 895-908, 2008.

[13] E. J. Allen and H. D. Victory Jr., "Modelling and simulation of a schistosomiasis infection with biological control," Acta Tropica, vol. 87, no. 2, pp. 251-267, 2003. 




Advances in

Operations Research

mansans

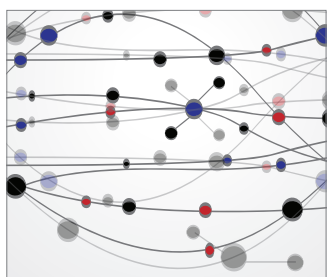

The Scientific World Journal
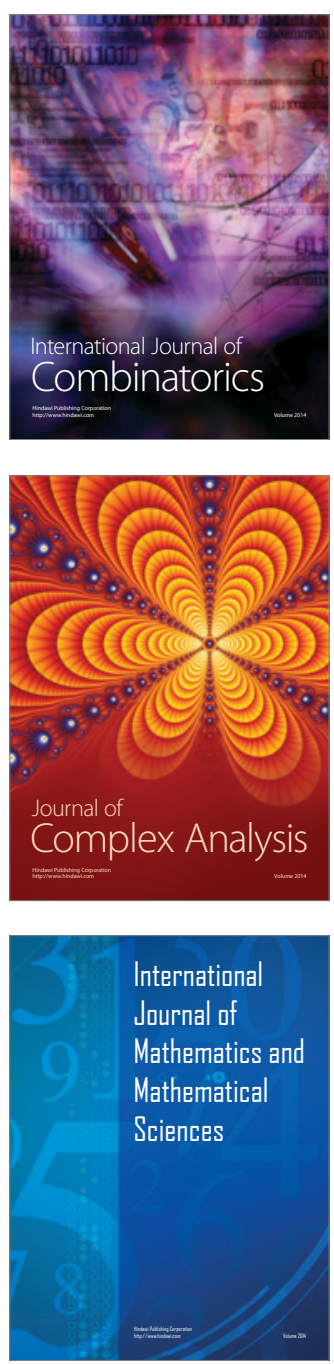
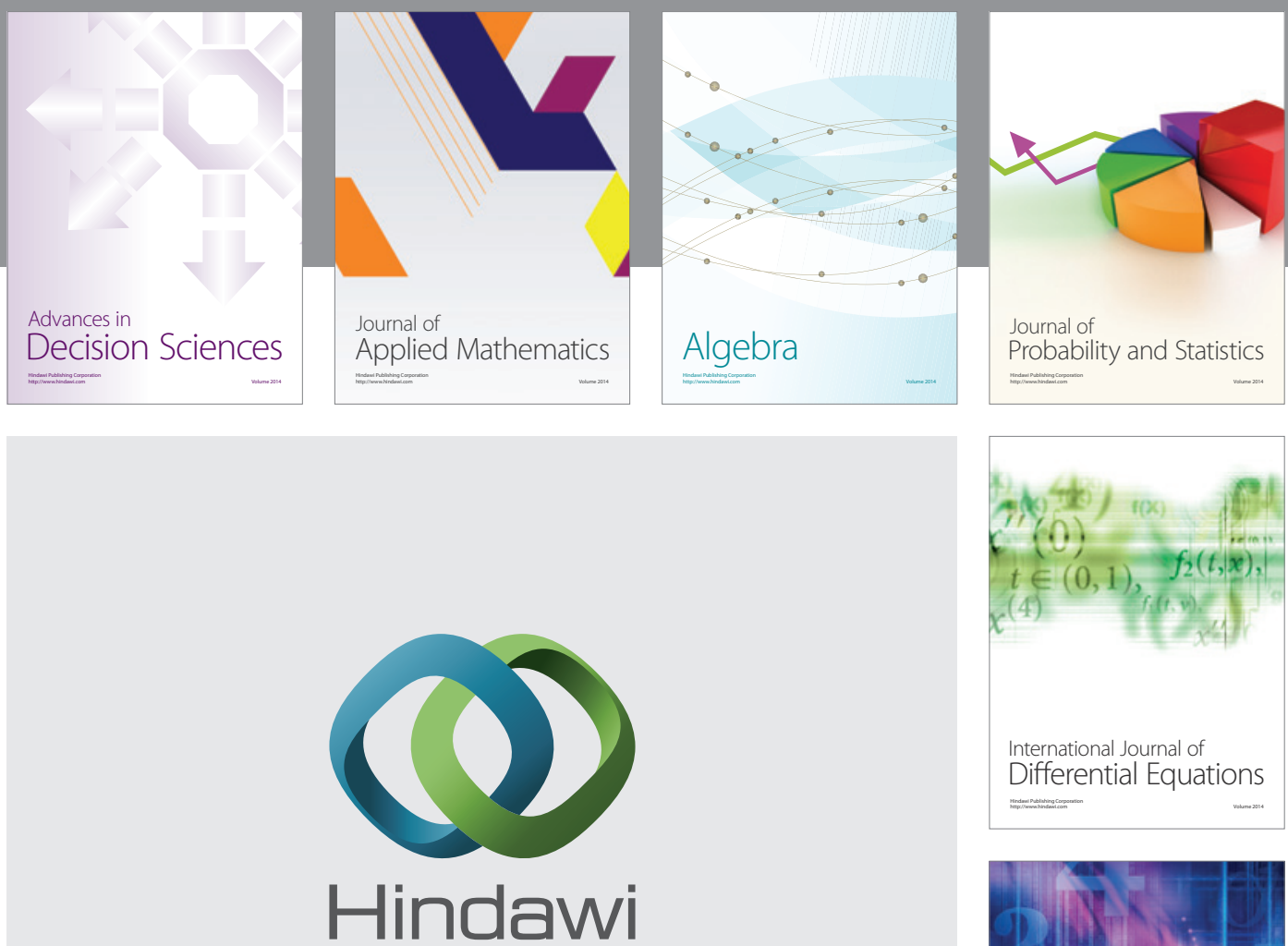

Submit your manuscripts at http://www.hindawi.com


Journal of

Function Spaces

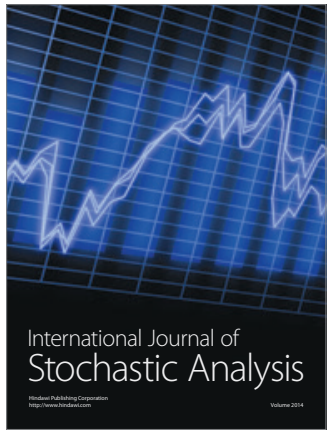

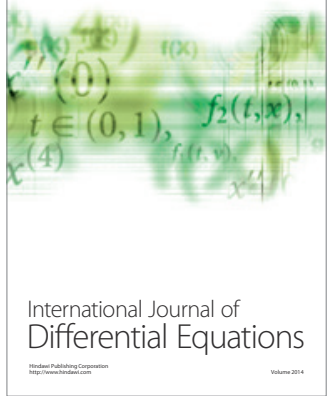
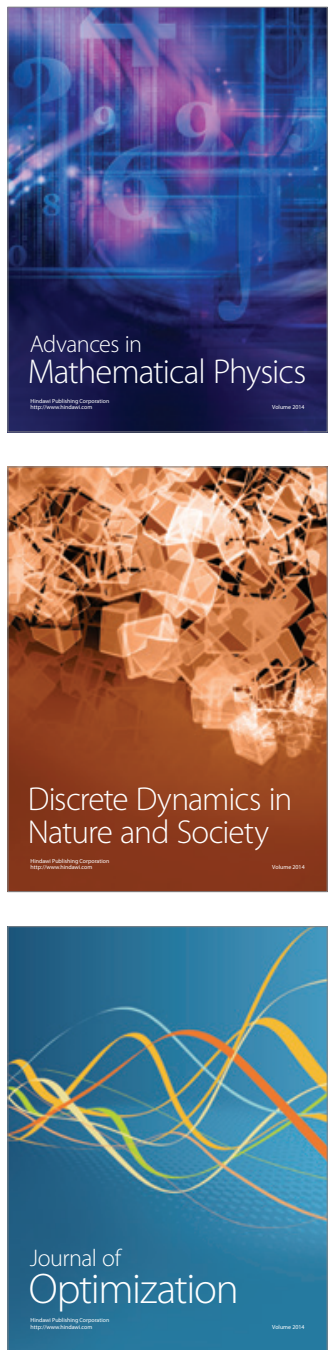